\title{
Greater Loss of White Matter Integrity in Postural Instability and Gait Difficulty Subtype of Parkinson's Disease
}

\author{
Quanquan Gu, Peiyu Huang, Min Xuan, Xiaojun Xu, Dan Li, Jianzhong Sun, \\ Hualiang Yu, Chao Wang, Wei Luo, Minming Zhang
}

\begin{abstract}
Background: Patients with the postural instability and gait difficulty (PIGD) subtype of Parkinson disease (PD) are at a higher risk of dysfunction and are less responsive to dopamine replacement therapy. The PIGD subtype was found to largely associate with white matter lesions, but details of the diffusion changes within these lesions have not been fully investigated. Voxel-based analysis for diffusion tensor imaging data is one of the preferred measures to compare diffusion changes in each voxel in any part of the brain. Methods: PD patients with the PIGD $(n=12)$ and non-PIGD subtypes $(n=12)$ were recruited to compare diffusion differences in fractional anisotropy, axial diffusivity, and radial diffusivity with voxel-based analysis. Results: Significantly reduced fractional anisotropy in bilateral superior longitudinal fasciculus, bilateral anterior corona radiata, and the left genu of the corpus callosum were shown in the PIGD subtype compared with the non-PIGD subtype. Increased radial diffusivity in the left superior longitudinal fasciculus was found in the PIGD subtype with no statistical differences in axial diffusivity found. Conclusions: Our study confirms previous findings that white matter abnormalities were greater in the PIGD subtype than in the non-PIGD subtype. Additionally, our findings suggested: (1) compared with the non-PIGD subtype, loss of white matter integrity was greater in the PIGD subtype; (2) bilateral superior longitudinal fasciculus may play a critical role in microstructural white matter abnormalities in the PIGD subtype; and (3) reduced white matter integrity in the PIGD subtype could be mainly attributed to demyelination rather than axonal loss.
\end{abstract}

RÉSUMÉ: Perte plus importante de l'intégrité de la substance blanche dans le sous-type PIGD de la maladie de Parkinson. Contexte: Des patients ayant un sous-type de la maladie de Parkinson (MP) associant une instabilité posturale et des difficultés à la marche (PIGD) présentent des risques plus élevés de troubles fonctionnels et réagissent moins au traitement substitutif par la dopamine. On a constaté que le sous-type PIGD était largement associé à des lésions de la substance blanche, mais les détails sur les modifications de la diffusion au sein de ces lésions n'ont pas encore été complètement étudiés. Une analyse des voxels pour les données d'imagerie du tenseur de diffusion est l'une des mesures préférentielles pour la comparaison des changements de diffusion dans chaque voxel, dans quelques régions du cerveau que ce soit. Méthodes: Des patients atteints de MP avec le sous-type PIGD $(n=12)$ et sans le sous-type PIGD ( $n=12)$ ont été recrutés pour comparer les différences de diffusion de l'anisotropie fractionnaire, de la diffusivité axiale et de la diffusivité radiale au moyen d'une analyse basée sur les voxels. Résultats: Une diminution significative de l'anisotropie fractionnaire a été constatée de chaque côté dans le faisceau longitudinal supérieur et dans la Corona radiata antérieure, ainsi que dans le genou du corps calleux du côté gauche chez les patients atteints du sous-type PIGD par rapport aux patients du soustype non-PIGD. L'augmentation de la diffusivité radiale du faisceau longitudinal supérieur gauche a été constatée dans le sous-type PIGD, sans qu'une différence statistique ait été constatée dans la diffusivité axiale. Conclusions: Notre étude confirme les constatations précédentes selon lesquelles les anomalies de la substance blanche sont plus importantes dans le sous-type PIGD que dans le sous-type non-PIGD. De plus, nos constatations suggèrent que : (1) la perte d'intégrité de la substance blanche a été plus importante dans le sous-type PIGD que dans le sous-type non-PIGD; (2) le faisceau longitudinal supérieur bilatéral pourrait jouer un rôle essentiel dans les anomalies microstructurelles de la substance blanche dans le sous-type PIGD; et (3) la diminution de l'intégrité de la substance blanche dans le sous-type PIGD pourrait être principalement attribué à la démyélinisation plutôt qu'à une perte axonale.

Keywords: Parkinson disease, neuroimaging, diffusion tensor imaging

doi:10.1017/cjn.2014.34

Can J Neurol Sci. 2014; 41: 763-768

Parkinson disease (PD) is the second most common neurodegenerative disease after Alzheimer disease and affects $1 \%$ of the population aged older than 65 years worldwide. ${ }^{1}$ Based on distinct clinical symptoms, PD patients can be divided into three subtypes: the postural instability and gait difficulty (PIGD) subtype, the tremor-dominant (TD) subtype, and the mixed subtype. ${ }^{2}$ Patients with the PIGD subtype present with predominant motor disorders such as falling, freezing of gait (FOG), and postural instability. Although patients with PIGD symptoms account for only $16 \%$ of all PD patients, ${ }^{3,4}$ these patients show significantly shorter life expectancy, worse prognosis, a higher risk of dementia and other nonmotor deficits. ${ }^{5}$ Furthermore, patients with the PIGD subtype are less responsive to routine dopamine replacement therapy, ${ }^{6}$ and

From the Department of Radiology (QG, PH, MX, XX, JS, CW, MZ), the Second Affiliated Hospital, Zhejiang University School of Medicine, Hangzhou, China; Department of Neurology (DL, WL), the Second Affiliated Hospital, Zhejiang University School of Medicine, Hangzhou, China; Department of Psychiatry (HY), the Second Affiliated Hospital, Zhejiang University School of Medicine, Hangzhou, China. Received June 17, 2014. Final Revisions Submitted September 2, 2014. Correspondence to: Minming Zhang, Department of Radiology, the Second Affiliated Hospital, Zhejiang University School of Medicine, Hangzhou, 310009, China. Email: zhangminming@zju.edu.cn 
surgical stimulation does not alleviate PIGD symptoms in the long term. ${ }^{7}$

Although PD has been widely recognized to result from the death of dopamine-generating cells in the substantia nigra, ${ }^{8}$ the major symptoms vary from person to person, indicating that $\mathrm{PD}$ is not a homogeneous entity ${ }^{9}$ and that multiple pathological processes are responsible for these different phenotypes. In recent years, a growing number of studies on the PIGD subtype have suggested that white matter lesions (WMLs), most of which are in periventricular and deep white matter regions of the brain, could be the potential underlying mechanism for the PIGD subtype. ${ }^{10-13}$ Compared with the non-PIGD subtype, the PIGD subtype shows more severe white matter damage on brain imaging ${ }^{10}$; however, patients with symptoms of tremor, rigidity, or other pure movement instability do not show the same white matter damage. ${ }^{13}$ Although these prior findings have suggested white matter damage is more relevant in the PIGD subtype than in the nonPIGD subtype, more research is needed.

Several investigations, many of which employed a visual method to assess the burden of WMLs on T2 fluid-attenuated inversion recovery (FLAIR)-based MRI, ${ }^{10-12}$ have studied the association between either WMLs or white matter hyperintensities and postural deficits and/or gait disturbances in PD. However, there are some disadvantages of this T2-FLAIR-based approach as follows: (1) some subtle white matter changes could be overlooked because of researchers' focus on leukoaraiosis ${ }^{11}$ and (2) the ceiling effect is hard to avoid and affects these visually estimated results. ${ }^{14}$ Although the T2-FLAIR-based method has benefited clinicians by allowing them to evaluate WMLs, diffusion properties can provide more informative details about WMLs, including which alterations have occurred before WMLs can be observed in T2-FLAIR images. To date, little attention has been paid either to microstructural white matter abnormalities or to forms of white matter degeneration in distinct phenotypes of PD.

Diffusion tensor imaging (DTI) is a noninvasive technique that provides a novel means of studying the microstructural integrity of white matter tracts. ${ }^{15}$ With diffusion indices of fractional anisotropy (FA), axial diffusivity (Da), and radial diffusivity (Dr) (calculated as the mean of the secondary and tertiary eigenvalues), ${ }^{16}$ the normal-appearing white matter foci can be evaluated and the form and the principal forms accounting for WMLs can be revealed. FA is the most commonly used index to reflect the degree of diffusion anisotropy as well as the integrity or disintegration of white matter tracts. ${ }^{17}$ The measure of Da stands for the diffusivity along the principal fiber axis and is more specific to axonal $\operatorname{loss}^{16}$; the measure of Dr represents the freedom of water molecules to travel across the principal axis and reflects damage to surrounding myelin sheaths. ${ }^{16}$ Voxel-based analysis (VBA) is one of the most preferred statistical methods to analyze the changes of diffusion measurements in each voxel in any part of the whole brain without a prior hypothesis, and has been used in both normal and diseased brains to compare DTI data in each voxel. ${ }^{18,19}$

In the present study, VBA of DTI data was performed to investigate white matter changes in global diffusion indices between the PIGD and non-PIGD subtypes of PD. We assumed the PIGD subtype had more severe microstructural white matter abnormalities compared with the non-PIGD subtype. Furthermore, by analyzing diffusion behaviors in the two subtypes, a better understanding of mechanisms underlying phenotypic variants and structural alterations in PD can be obtained.

\section{Materials AND Methods \\ Patients}

All of this study's patients were recruited from the department of neurology at the 2nd Affiliated Hospital of Zhejiang University and diagnosed with PD according to the UK Parkinson's Disease Society Brain Bank criteria for idiopathic PD ${ }^{20}$ Disease severity was evaluated using the Unified Parkinson's Disease Rating Scale and the Hoehn and Yahr scale. The Mini-Mental State Examination was used to screen for cognitive dysfunction and ensure patient compliance with MRI scanning. All recruited patients were eligible for MRI scanning; underwent clinical interviews in person to ensure the absence of any history of brain traumas, psychiatric diseases, or self-reported cardio-cerebral accidents; and were able to complete independent ambulation without walking aids. The disease duration was defined as the period from when the patient could recognize PD symptoms to when he or she participated in the study. According to the ratio of mean tremor score/mean PIGD score, ${ }^{2}$ each PD patient was grouped into the PIGD subtype $(\leq 1.0)$ or the non-PIGD subtype $(>1.0$ or mean PIGD score $=0$ ). Subscores for rigidity (item 22), bradykinesia (items 23-26, 31), tremor (items 16, 20, 21), and PIGD (items $13-15,29,30$ ) in Unified Parkinson's Disease Rating Scale were also collected. In addition, the levodopa equivalent dose was calculated for each patient according to the conversion formula provided by a systematic review. ${ }^{21}$

A total of $24 \mathrm{PD}$ patients (female/male $=9 / 15)$ were recruited and divided into the PIGD subtype (12 patients, female/male $=4 / 8$ ) and the non-PIGD subtype (12 patients, female/male $=5 / 7)$. Of the 24 patients, 8 were recently diagnosed and had not gone through any treatment, whereas the other 16 patients who had been medically treated and were required to follow a 12-hour withdrawal from medication. All recruited patients provided written informed consent. Approval from the medical ethics committee of the hospital was obtained for this study.

\section{MR Data Acquisition}

The DTI scans were performed using a 3.0 T GE Signa EXCITE MR scanner equipped with an eight-channel head coil. Images were acquired in the axial plane. An echo-planar imaging sequence with 15 noncollinear diffusion sensitization gradients $\left(b=1000 \mathrm{~s} / \mathrm{mm}^{2}\right)$ and another nonweighted diffusion image $\left(\mathrm{b} 0=0 \mathrm{~s} / \mathrm{mm}^{2}\right.$ ) was performed with parameters as follows: repetition time/echo time $=2000 / 30 \mathrm{~ms}$; field of view $=240 \times 240 \mathrm{~mm}^{2}$; acquisition matrix $=128 \times 128,38$ slices, slice thickness $=3 \mathrm{~mm}$, and flip angle $=90^{\circ}$. All recruited patients were instructed to keep their eyes closed but not to fall asleep and relax their bodies but keep their heads immobile during scanning. Foam padding and earplugs were used to reduce head motion and scanner noise.

\section{Image Preprocessing}

The workflow of image preprocessing was as follows: the freshly obtained image data were converted to a three-dimensional NIfTI hdr/img. Using DTIstudio (http://cmrm.med.jhmi.edu/, version 2.4.01), the eddy currents and motion distortions were corrected for each subject via a linear transformation approach in automatic image registration. The diffusion tensors for each voxel were then calculated to yield images for FA, $\operatorname{Da}\left(\lambda_{\|}\right)$, and $\operatorname{Dr}\left(\lambda_{\perp}\right)$. 


\section{Image Analysis}

Before analyses, all images for FA, Da, and Dr were normalized using the parameters obtained from the normalization of the $\mathrm{b}=0$ image to an MNI template (McGill University, Montreal, QC, Canada) in SPM8 (Wellcome Trust Centre for Neuroimaging, London, UK) based on the MATLAB R2009b platform (MathWorks Inc, Natick, MA, USA). All images were resampled into a voxel size of $2 \times 2 \times 2 \mathrm{~mm}^{3}$. The normalized images were smoothed with an 8-mm full-width at half-maximum Gaussian kernel to minimize space noise.

VBA for global white matter differences of the two subtypes was performed in SPM8. A white matter mask (voxel size $2 \times 2 \times 2 \mathrm{~mm}^{3}$ ) was applied in MNI space from the DPARSF package $^{22}$ for statistical analysis. An unpaired two-sample t test was performed using the smoothed images of FA, Da, and Dr. As stated in the Results section, disease duration was different between the two groups, thus it was set as a covariate in the statistical test. Individual voxel and uncorrected significance cutoff were set at $\mathrm{p}<0.001$, cluster size $>10$ voxels. We considered cluster sizes larger than 10 voxels and corrected family-wise error (FWE) at $\mathrm{p}<.05$ to be significant after correction for multiple comparisons. The obtained statistical t-maps were overlaid on an MNI template of a T1-weighted image. In addition, we used the Fazekas scale, which is one of the most popular visual rating scales, ${ }^{23}$ to rate white matter changes visible on FLAIR-T2W images for each subject (see Supplementary Table 3).

Demographic variables between the two subtypes were calculated using SPSS software (v.16.0; Chicago, IL. USA). Because of the sample size, the unpaired Student's t test and the Fisher's exact test were chosen, with the statistical threshold at $\mathrm{p}<0.05$.

\section{RESULTS}

Demographic characteristics of the PIGD and non-PIGD subtypes are summarized in Table 1 and Supplementary Tables 1 and 2. There were no significant differences between the two subtypes in their clinical details except for disease duration and tremor score. White matter changes visible on FLAIR-T2W images did not differ between the two subtypes (Supplementary Table 3).

In the chosen test design $\left(\mathrm{p}_{\text {unc }}<0.001\right.$, cluster size $>10$ voxels), FA reductions in the PIGD subtype were shown in the areas of bilateral superior longitudinal fasciculus (SLF, $\mathrm{p}_{\text {unc- } \mathrm{L}}=$ 0.000017, 0.000047; $\mathrm{p}_{\text {unc-R }}=0.000082$ ), bilateral anterior corona
Table 1: Clinical characteristics of all the recruited patients

\begin{tabular}{l|c|c|c|c|c}
\hline & All PD & PIGD & Non-PIGD & t value & p \\
\hline Age (y) & $55.9 \pm 8.1$ & $55.7 \pm 8.1$ & $56.0 \pm 8.4$ & -0.07 & 0.942 \\
\hline Female/male & $9 / 15$ & $4 / 8$ & $5 / 7$ & - & $1.000^{*}$ \\
\hline Duration (y) & $5.7 \pm 4.4$ & $3.2 \pm 3.1$ & $8.0 \pm 3.8$ & -3.43 & $\mathbf{0 . 0 0 2} \dagger$ \\
\hline H\&Y & $2.5 \pm 0.7$ & $2.5 \pm 0.8$ & $2.5 \pm 0.7$ & -0.14 & 0.892 \\
\hline MMSE & $27.5 \pm 1.6$ & $27.3 \pm 1.6$ & $27.6 \pm 1.6$ & -0.38 & 0.709 \\
\hline UPDRS & $45.5 \pm 20.5$ & $40.7 \pm 17.4$ & $50.3 \pm 22.9$ & -1.15 & 0.261 \\
\hline UPDRS III & $31.8 \pm 14.9$ & $29.2 \pm 12.5$ & $34.4 \pm 17.1$ & -0.85 & 0.407 \\
\hline PIGD & $3.7 \pm 1.8$ & $3.7 \pm 1.8$ & $3.7 \pm 1.8$ & -0.11 & 0.912 \\
\hline Rigidity & $8.4 \pm 4.9$ & $9.3 \pm 4.9$ & $7.5 \pm 4.9$ & 0.91 & 0.371 \\
\hline Bradykinesia & $13.6 \pm 5.7$ & $13.3 \pm 6.00$ & $13.9 \pm 5.6$ & -0.25 & 0.807 \\
\hline
\end{tabular}

Values are expressed as mean \pm standard deviation. Numerical data were compared by unpaired Student's t tests and crosstab chi-square tests.

$\mathrm{H} \& \mathrm{Y}=$ Hoehn and Yahr scale; MMSE = Mini-Mental State Examination; UPDRS = Unified Parkinson's Disease Rating Scale.

*Contrast evaluated using two-tailed Fisher's exact test.

$\dagger$ The statistical difference between the PIGD and non-PIGD subtypes with the statistical threshold set at $\mathrm{p}<0.05$.

radiata $\left(A C R, p_{u n c-L}=0.000655 ; p_{\text {unc- } \mathrm{R}}=0.000144\right)$ and the left genu of the corpus callosum $\left(\mathrm{GCC}, \mathrm{p}_{\text {unc-L }}=0.000311\right)$. Using FWE-corrected $\mathrm{p}<0.05$, there were clusters of significant FA reductions in bilateral superior longitudinal fasciculus $\left(\mathrm{p}_{\text {fwe-L }}=0.011,0.025 ; \mathrm{p}_{\text {fwe-R }}=0.039\right)$. There was no any statistical difference in Da between the two subtypes, either corrected or uncorrected. In the analysis of the level of Dr, a slightly higher level of Dr of the left SLF was found in PIGD subtype compared with the non-PIGD subtype $\left(\mathrm{p}_{\text {unc- } \mathrm{L}}=0.000425\right)$ using an uncorrected threshold $\left(\mathrm{p}_{\text {unc }}<0.001\right.$, cluster size $\left.>10\right)$. (Figure 1; Tables 2 and 3)

\section{DISCUSSION}

The current study investigated differences in microstructural white matter abnormalities in PIGD and non-PIGD subtypes of PD using a voxel-based analytic approach for DTI data. Measurements of FA, Da, and Dr were used to assess the degree of microstructural white matter abnormalities. As a whole, after

Table 2: The FA analysis of the PIGD subtype compared with the non-PIGD subtype

\begin{tabular}{|c|c|c|c|c|c|c|}
\hline Region & Cluster size & $\mathbf{X}$ & $\mathbf{Y}$ & $\mathbf{Z}$ & Peak T & $\mathbf{p}_{\text {uncor }}\left(\mathbf{p}_{\text {fwe }}\right)$ \\
\hline Superior longitudinal fasciculus (L) & 458 & -32 & -10 & 26 & -5.24 & $0.000017(\mathbf{0 . 0 1 1} *)$ \\
\hline Superior longitudinal fasciculus $(\mathrm{L})$ & 62 & -36 & -48 & 24 & -4.81 & $0.000047(\mathbf{0 . 0 2 5} *)$ \\
\hline Superior longitudinal fasciculus (R) & 186 & 30 & -36 & 34 & -4.57 & $0.000082(\mathbf{0 . 0 3 9} *)$ \\
\hline Anterior corona radiata $(\mathbf{R})$ & 13 & 20 & 34 & 22 & -4.34 & $0.000144(0.061)$ \\
\hline Genu of the corpus callosum (L) & 15 & -16 & 32 & 12 & -4.02 & $0.000311(0.110)$ \\
\hline Anterior corona radiata $(L)$ & 10 & -24 & 32 & 10 & -3.71 & $0.000655(0.189)$ \\
\hline
\end{tabular}

Disease duration was set as a covariate. The uncorrected threshold $\left(\mathrm{p}_{\text {uncor }}\right)$ is at $\mathrm{p}<0.001$ and cluster size $>10$ voxels.

$* P$ indicates family-wise error-corrected results $\left(\mathrm{p}_{\mathrm{fwe}}\right)$ at $\mathrm{p}<0.05$, cluster size $>10$ voxels. Results are displayed in the MNI coordinates.

$\mathrm{L}=$ left; $\mathrm{R}=$ right. 
Table 3: Dr analysis of the PIGD subtype compared with the non-PIGD subtype

\begin{tabular}{l|c|c|c|c|c|c}
\hline Region & Cluster size & $\mathbf{X}$ & $\mathbf{Y}$ & $\mathbf{Z}$ & Peak T & $\mathbf{p}_{\text {uncor }}\left(\mathbf{p}_{\text {fwe }}\right)$ \\
\hline Superior longitudinal fasciculus (L) & 18 & -36 & -48 & 24 & 3.89 & $0.000425(0.124)$ \\
\hline
\end{tabular}

Disease duration was set as a covariate. The uncorrected threshold $\left(\mathrm{p}_{\text {uncor }}\right.$ ) is at $\mathrm{p}<0.001$ and cluster size $>10$ voxels. No clusters survive after FWE correction. Results are displayed in the MNI coordinates.

$\mathrm{L}=$ left.

controlling disease duration, between-group analyses showed much more severe microstructural white matter impairments in the PIGD subtype compared with the non-PIGD subtype. Significant FA reductions were shown in the bilateral SLF and bilateral ACR areas as well as the left GCC in the PIGD subtype compared with the non-PIGD subtype. Moreover, a marked Dr increase was shown in the left SLF in the PIGD subtype without a difference in Da. Furthermore, our study suggested that the principal cause of these WMLs in the PIGD subtype could be demyelination of sheath surrounding the axon rather than disintegration of the axonal skeleton.

Posture and gait are an interconnected issue. There is renewed interest in the effects of PD on the abnormalities of gait and posture. Recent work indicated extensive involvement of extra-nigral structures were also involved in PIGD symptoms of PD-for example, the frontal-parietal lobes, the reticular formation, and some brainstem nuclei such as the mesencephalic locomotor region and in particular, the pedunculopontine nucleus $(\mathrm{PPN}) .{ }^{24-26} \mathrm{PD}$ patients tend to fall, with the underlying reason being that they are less capable of controlling their forward posture in the forward-backward direction than in the left-right direction. ${ }^{27}$ Festination and freezing as well as falling when posture changes are thought to be influenced by deficits in vestibular, proprioceptive, and attention functions in $\mathrm{PD} .^{28-31}$
The SLF is regarded as the connections between frontal, parietal, temporal, and occipital lobes in human, which can be divided into the SLF I (dorsal), the SLF II (middle), and the SLF III (ventral) and the arcuate fascicle. ${ }^{32}$ The projections of the SLF overlap with extensive brain areas. First, these projections are associated with the dorsal and ventral components of the frontoparietal network, which mediates visuospatial processing in both goal-directed and stimulus-driven attention. ${ }^{33}$ In addition, the SLF overlaps with the course of the cortico-pontine-cerebellar circuits (part of the extrapyramidal system), from the premotor cortex, precentral gyrus (the motor strip), and postcentral gyrus (the sensory strip) to the cerebellum via the pontine nuclei. The circuits allow the cerebellum to assist cortico-bulbar and cortico-spinal tracts in regulating voluntary movements initiated from the cortices. ${ }^{34}$ Therefore, SLF lesions will disrupt information transmitted through cortico-cortical or corico-pontine-cerebellar circuits.

In terms of cortico-cortical circuits, many neuroimaging studies have investigated posture and gait. A functional resting-state study $^{35}$ reported that PD patients with gait disturbances had significantly reduced functional connectivity in both "executiveattention" (in the right middle frontal gyrus and in the angular gyrus) and visual (in the right occipito-temporal gyrus) networks. Moreover, the severity of FOG was significantly correlated with

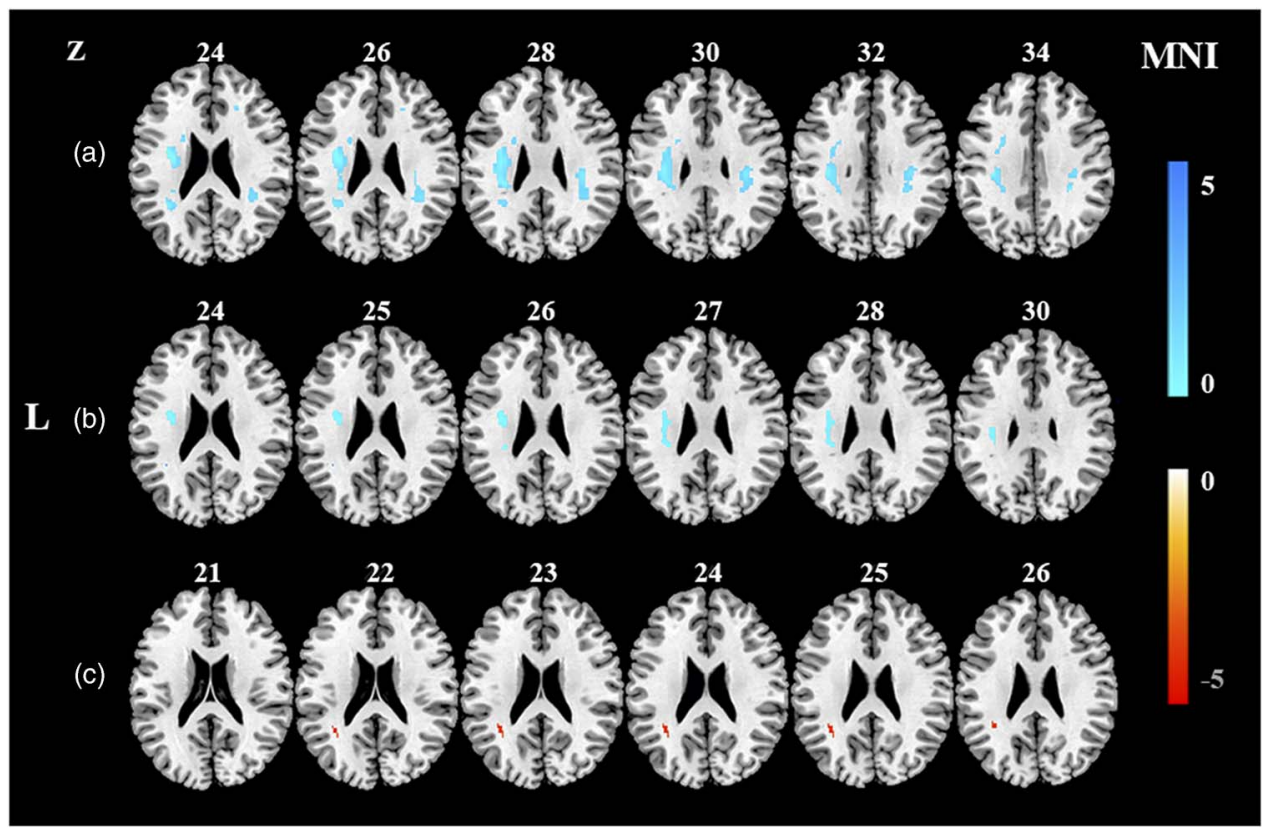

Figure 1: Comparisons of diffusion indices in the PIGD subtype versus the non-PIGD subtype. (A) The PIGD subtype showed reduced FA in the bilateral $S L F$, bilateral anterior corona radiata, and the left genu of the corpus callosum (punc $<0.001$, cluster size $>10$ ). (B) Using a FWE correction (pfwe $<0.05$, cluster size > 10), only clusters in the bilateral SLF with pronounced FA reductions survived. (C) Increased Dr was found in the left SLF in PIGD versus the non-PIGD subtype (punc $<0.001$, cluster size $>10$ ). Results are displayed in the MNI coordinates. FWE $=$ family-wise error; $L=$ left. 
decreased connectivity within the two networks. ${ }^{35}$ Other evidence $^{36,37}$ suggested that changes in cognition and environment also acted on motor disorders such as FOG, which often arise in gait initiation, turning, walking through narrow corridors, changes in surroundings, and even when the individual is anxious or under pressure. In terms of cortico-pontine-cerebellar circuits, Schweder et al. ${ }^{38}$ characterized the PPN connectivity profiles of PD patients with and without FOG as well as healthy controls. ${ }^{38}$ They found connectivity between the PPN and the cerebellum in controls and non-FOG PD, whereas FOG patients showed an absence of cerebellar connectivity and increased visibility of the decussation of cortico-pontine fibers in the anterior pons. Their findings stressed the importance of cortico-pontine-cerebellar circuits in the pathophysiology of this gait disorder. ${ }^{38}$ A study led by Rosenberg-Katz et al. ${ }^{25}$ has shown apparent gray matter atrophy and decreased functional connectivity in motor-related regions in the PIGD subtype in contrast to TD subtype, suggesting gray matter atrophy in cortical and subcortical motor-planning areas may partly account for PIGD symptoms in PD.

The GCC is the anterior segment of the corpus callosum (CC) that receives projection from the prefrontal cortical region ${ }^{39}$ and is associated with premotor and cognition functions. In a normal older population, an independent relationship between gait and genual CC abnormalities has been found. ${ }^{40}$ Similarly, compared with general PD, the GCC was more affected in progressive supranuclear palsy with significantly reduced FA values, which was one of the most common forms of atypical parkinsonian syndromes. $^{41}$ In addition, another DTI study ${ }^{42}$ on diffusion alterations in the PIGD subtype showed lower FA values in the genu and the body of the CC compared with controls, providing evidence that abnormalities in the $\mathrm{CC}$ are involved in gait disorders. These findings all support the important role of the $\mathrm{CC}$ in the pathogenic process of the PIGD subtype.

In addition to the SLF and the GCC, the ACR was another area with decreased FA value in PIGD subtype in our results. The ACR is considered to be an important white matter tract running adjacent to the anterior cingulate cortex, the striatum, and other structures. ${ }^{43}$ Projections of the ACR originate from the primary motor areas, the supplementary motor area, and anterior cingulated cortex to the brainstem reticular formation ${ }^{44}$; thus, motor control would be affected if the ACR were impaired. A lower ACR gray matter volume was observed in the PIGD subtype compared with the TD subtype. ${ }^{25}$ In addition, several DTI investigations using FA as a measure found the ACR was specifically related to executive attention. ${ }^{4,46}$ A single-photon emission CT scan study ${ }^{47}$ compared cerebral blood flow between patients with either the TD or PIGD subtype and controls and showed hypoperfusion within the anterior cingulate cortex in the PIGD subtype. This result was believed to be from overactivity of the thalamus, which made thalamo-cortical projections fail to communicate with the motor-related cortical areas (i.e. cingulated motor areas). ${ }^{48}$

To our knowledge, few studies have investigated Da or Dr in different subtypes of PD. In the present study, we found elevated Dr in the PIGD subtype, whereas the difference in Da did not reach statistical significance. Though demyelination is not a critical pathogenic feature in PD as a whole, ${ }^{49}$ the role of demyelination in different PD subtypes is still open to question. Changed Dr with unchanged Da normally suggests a marked loss of myelin. ${ }^{16,50}$ The compactness of the axonal fibers may alter according to alpha-synuclein accumulation and microglia activation, ${ }^{51}$ which are related to neuroinflammatory responses in the brain, ${ }^{52}$ thereby increasing Dr. ${ }^{53}$ In our study, only the left SLF showed increased Dr and reduced FA. In addition, absence of statistical significance shown in the Da did not demonstrate that axons were intact in the two subtypes. The probable explanation is the effect of axonal degeneration had not reached significance, but potential neural mechanisms await further investigation. Overall, the presence of increased Dr in the left SLF combined with reduced FA in the bilateral SLF indicates that demyelination could be a critical reason for the more severe WMLs of the PIGD subtype.

There are limitations of this study. First, the sample size was small and we compared PD subtypes only, without normal controls. Second, disease duration differed between the two subgroups, thereby influencing the comparability of the groups. Last, the effects of nigrostriatal dopaminergic denervation on white matter impairments in PD have not been considered (e.g. substantia nigra, striatal nuclei, levodopa dose equivalency).

\section{CONCLUSIONS}

Our study confirms more severe white matter damage in the PIGD subtype compared with the non-PIGD subtype of PD. Furthermore, our findings suggested the following: (1) compared with the non-PIGD subtype, patients with the PIGD subtype had more severe microstructural white matter abnormalities; (2) bilateral superior longitudinal fasciculus may play a critical role in white matter disintegration in the PIGD subtype; and (3) reduced white matter integrity in the PIGD subtype can be mainly attributed to demyelination rather than axonal loss.

\section{ACKNOWLEDgMents AND Funding}

This work was cofunded by the 12th Five-year Plan for National Science \& Technology Supporting Program of China (Grant No. 2012BAI10B04); the Natural Science Foundation of Zhejiang Province, China (Grant No. LY12H09006); and the National Natural Science Foundation of China (Grant Nos. 81301190 and 81371519).

\section{SuPPlementary Material}

To view supplementary material for this article, please visit http://dx.doi.org/10.1017/cjn.2014.34

\section{REFERENCES}

1. Tanner CM, Goldman SM. Epidemiology of Parkinson's disease. Neurol Clin. 1996;14(2):317-35.

2. Jankovic J, McDermott M, Carter J, et al. Variable expression of Parkinson's disease: a base-line analysis of the DATATOP cohort. The Parkinson Study Group. Neurology. 1990;40(10):1529-34.

3. Rajput AH, Pahwa R, Pahwa P, Rajput A. Prognostic significance of the onset mode in parkinsonism. Neurology. 1993;43(4): 829-30.

4. Tykocki T, Mandat T, Nauman P. Pedunculopontine nucleus deep brain stimulation in Parkinson's disease. Arch Med Sci. 2011; 7(4):555-64.

5. Auyeung M, Tsoi TH, Mok V, et al. Ten year survival and outcomes in a prospective cohort of new onset Chinese Parkinson's disease patients. J Neurol Neurosurg Psychiatry. 2012;83(6):607-11.

6. Bohnen NI, Cham R. Postural control, gait, and dopamine functions in parkinsonian movement disorders. Clin Geriatr Med. 2006; 22(4):797-812,vi. 
7. St George RJ, Nutt JG, Burchiel KJ, Horak FB. A meta-regression of the long-term effects of deep brain stimulation on balance and gait in PD. Neurology. 2010;75(14):1292-9.

8. Bohnen NI, Albin RL. The cholinergic system and Parkinson disease. Behav Brain Res. 2011;221(2):564-73.

9. Zetusky WJ, Jankovic J, Pirozzolo FJ. The heterogeneity of Parkinson's disease: clinical and prognostic implications. Neurology. 1985;35(4): 522-6.

10. Bohnen NI, Muller ML, Zarzhevsky N, et al. Leucoaraiosis, nigrostriatal denervation and motor symptoms in Parkinson's disease. Brain. 2011;134(Pt 8):2358-65.

11. Herman T, Rosenberg-Katz K, Jacob Y, et al. White matter hyperintensities in Parkinson's disease: do they explain the disparity between the postural instability gait difficulty and tremor dominant subtypes? PLoS One. 2013;8(1):e55193.

12. Lee SJ, Kim JS, Lee KS, et al. The severity of leukoaraiosis correlates with the clinical phenotype of Parkinson's disease. Arch Gerontol Geriatr. 2009;49(2):255-9.

13. Piccini P, Pavese N, Canapicchi R, et al. White matter hyperintensities in Parkinson's disease. Clinical correlations. Arch Neurol. 1995;52(2):191-4.

14. Gouw AA, van der Flier WM, van Straaten EC, et al. Reliability and sensitivity of visual scales versus volumetry for evaluating white matter hyperintensity progression. Cerebrovasc Dis. 2008;25(3): 247-53.

15. Le Bihan D, Mangin JF, Poupon C, et al. Diffusion tensor imaging: concepts and applications. J Magn Reson Imaging. 2001;13(4): 534-46.

16. Song SK, Sun SW, Ramsbottom MJ, Chang C, Russell J, Cross AH. Dysmyelination revealed through MRI as increased radial (but unchanged axial) diffusion of water. Neuroimage. 2002;17(3): 1429-36.

17. Beaulieu C. The basis of anisotropic water diffusion in the nervous system - a technical review. NMR Biomed. 2002; 15(7-8):435-55.

18. Ashburner J, Friston KJ. Voxel-based morphometry-the methods. Neuroimage. 2000;11(6 Pt 1):805-21.

19. Zhang K, Yu C, Zhang Y, et al. Voxel-based analysis of diffusion tensor indices in the brain in patients with Parkinson's disease. Eur J Radiol. 2011;77(2):269-73.

20. Hughes AJ, Daniel SE, Kilford L, Lees AJ. Accuracy of clinical diagnosis of idiopathic Parkinson's disease: a clinico-pathological study of 100 cases. J Neurol Neurosurg Psychiatry. 1992;55(3):181-4.

21. Tomlinson CL, Stowe R, Patel S, Rick C, Gray R, Clarke CE. Systematic review of levodopa dose equivalency reporting in Parkinson's disease. Mov Disord. 2010;25(15):2649-53.

22. Chao-Gan Y, Yu-Feng Z. DPARSF: A MATLAB Toolbox for "Pipeline" Data Analysis of Resting-State fMRI. Front Syst Neurosci. 2010;4:13.

23. Fazekas F, Chawluk JB, Alavi A, Hurtig HI, Zimmerman RA. MR signal abnormalities at $1.5 \mathrm{~T}$ in Alzheimer's dementia and normal aging. AJR Am J Roentgenol. 1987;149(2):351-6.

24. Grabli D, Karachi C, Welter ML, et al. Normal and pathological gait: what we learn from Parkinson's disease. J Neurol Neurosurg Psychiatry. 2012;83(10):979-85.

25. Rosenberg-Katz K, Herman T, Jacob Y, Giladi N, Hendler T, Hausdorff JM. Gray matter atrophy distinguishes between Parkinson disease motor subtypes. Neurology. 2013;80(16):1476-84.

26. Jahn K, Deutschlander A, Stephan $T$, et al. Imaging human supraspinal locomotor centers in brainstem and cerebellum. Neuroimage. 2008;39(2):786-92.

27. Heremans E, Nieuwboer A, Vercruysse S. Freezing of gait in Parkinson's disease: where are we now? Curr Neurol Neurosci Rep. 2013; 13(6):350.

28. Bloem BR, Grimbergen YA, Cramer M, Willemsen M, Zwinderman AH. Prospective assessment of falls in Parkinson's disease. J Neurol. 2001;248(11):950-8.

29. Jacobs JV, Horak FB, Van Tran K, Nutt JG. An alternative clinical postural stability test for patients with Parkinson's disease. J Neurol. 2006;253(11):1404-13.

30. Camicioli R, Oken BS, Sexton G, Kaye JA, Nutt JG. Verbal fluency task affects gait in Parkinson's disease with motor freezing. J Geriatr Psychiatry Neurol. 1998;11(4):181-5.
31. Bond JM, Morris M. Goal-directed secondary motor tasks: their effects on gait in subjects with Parkinson disease. Arch Phys Med Rehabil. 2000;81(1):110-6.

32. Makris N, Kennedy DN, McInerney S, et al. Segmentation of subcomponents within the superior longitudinal fascicle in humans: a quantitative, in vivo, DT-MRI study. Cereb Cortex. 2005; 15(6):854-69.

33. Corbetta M, Shulman GL. Control of goal-directed and stimulusdriven attention in the brain. Nat Rev Neurosci. 2002;3(3): 201-15.

34. Leergaard TB, Bjaalie JG. Topography of the complete corticopontine projection: from experiments to principal Maps. Front Neurosci. 2007;1(1):211-23.

35. Tessitore A, Amboni M, Esposito F, et al. Resting-state brain connectivity in patients with Parkinson's disease and freezing of gait. Parkinsonism Relat Disord. 2012;18(6):781-7.

36. Schaafsma JD, Balash Y, Gurevich T, Bartels AL, Hausdorff JM, Giladi N. Characterization of freezing of gait subtypes and the response of each to levodopa in Parkinson's disease. Eur J Neurol. 2003;10(4):391-8.

37. Giladi N, McMahon D, Przedborski S, et al. Motor blocks in Parkinson's disease. Neurology. 1992;42(2):333-9.

38. Schweder PM, Hansen PC, Green AL, Quaghebeur G, Stein J, Aziz TZ. Connectivity of the pedunculopontine nucleus in parkinsonian freezing of gait. Neuroreport. 2010;21(14):914-6.

39. Witelson SF. Hand and sex differences in the isthmus and genu of the human corpus callosum. A postmortem morphological study. Brain. 1989;112(Pt 3):799-35.

40. Bhadelia RA, Price LL, Tedesco KL, et al. Diffusion tensor imaging, white matter lesions, the corpus callosum, and gait in the elderly. Stroke. 2009;40(12):3816-20.

41. Ito S, Makino T, Shirai W, Hattori T. Diffusion tensor analysis of corpus callosum in progressive supranuclear palsy. Neuroradiology. 2008;50(11):981-5.

42. Chan LL, Ng KM, Rumpel H, Fook-Chong S, Li HH, Tan EK. Transcallosal diffusion tensor abnormalities in predominant gait disorder parkinsonism. Parkinsonism Relat Disord. 2014;20(1): 53-9.

43. Wakana S, Jiang H, Nagae-Poetscher LM, van Zijl PC, Mori S. Fiber tract-based atlas of human white matter anatomy. Radiology. 2004;230(1):77-87.

44. Hanakawa T, Katsumi Y, Fukuyama H, et al. Mechanisms underlying gait disturbance in Parkinson's disease: a single photon emission computed tomography study. Brain. 1999;122(Pt 7): 1271-82.

45. Niogi S, Mukherjee P, Ghajar J, McCandliss BD. Individual differences in distinct components of attention are linked to anatomical variations in distinct white matter tracts. Front Neuroanat. 2010;4:2.

46. Niogi SN, Mukherjee P, Ghajar J, et al. Structural dissociation of attentional control and memory in adults with and without mild traumatic brain injury. Brain. 2008;131(Pt 12):3209-21.

47. Mito Y, Yoshida K, Yabe I, et al. Brain SPECT analysis by 3D-SSP and phenotype of Parkinson's disease. J Neurol Sci. 2006; 241(1-2):67-72.

48. Playford ED, Jenkins IH, Passingham RE, Nutt J, Frackowiak RS, Brooks DJ. Impaired mesial frontal and putamen activation in Parkinson's disease: a positron emission tomography study. Ann Neurol. 1992;32(2):151-61.

49. Teismann P, Schulz JB. Cellular pathology of Parkinson's disease: astrocytes, microglia and inflammation. Cell Tissue Res. 2004;318(1):149-61.

50. Klawiter EC, Schmidt RE, Trinkaus K, et al. Radial diffusivity predicts demyelination in ex vivo multiple sclerosis spinal cords. Neuroimage. 2011;55(4):1454-60.

51. Smith KJ, Blakemore WF, McDonald WI. The restoration of conduction by central remyelination. Brain. 1981;104(2):383-404.

52. Ouchi Y, Yoshikawa E, Sekine Y, et al. Microglial activation and dopamine terminal loss in early Parkinson's disease. Ann Neurol. 2005;57(2):168-75.

53. Theilmann RJ, Reed JD, Song DD, et al. White-matter changes correlate with cognitive functioning in Parkinson's disease. Front Neurol. 2013;4:37. 